\title{
TRACE: A Stigmergic Crowdsourcing Platform for Intelligence Analysis
}

\author{
Huichuan Xia, Carsten Østerlund, Brian McKernan, James Folkestad, Patricia Rossini, Olga Boichak, \\ Jerry Robinson, Kate Kenski, Roc Myers, Benjamin A. Clegg, Jennifer Stromer-Galley
}

\begin{abstract}
Crowdsourcing has become a frequently adopted approach to solving various tasks from conducting surveys to designing products. In the field of reasoning-support, however, crowdsourcing-related research and application have not been extensively implemented. Reasoning-support is essential in intelligence analysis to help analysts mitigate various cognitive biases, enhance deliberation, and improve report writing. In this paper, we propose a novel approach to designing a crowdsourcing platform that facilitates stigmergic coordination, awareness, and communication for intelligence analysis. We have partly materialized our proposal in the form of a crowdsourcing system which supports intelligence analysis: TRACE (Trackable Reasoning and Analysis for Collaboration and Evaluation). We introduce several stigmergic approaches integrated into TRACE and discuss the potential experimentation of these approaches. We also explain the design implications for further development of TRACE and similar crowdsourcing systems to support reasoning.
\end{abstract}

\section{Introduction}

Crowdsourcing platforms depend on two principle types of data to be productive: direct contributions from the crowd members; and opportunistic or mediated data such as data automatically collected by a wearable application and those collected transmitted via an intermediary platform like Amazon Mechanical Turk (MTurk). To ensure the quality of these contributions, crowdsourcing platforms need to support crowd workers' reasoning and decisionmaking in a way that does not overwhelm them with extrinsic cognitive load or lead them to unintended cognitive biases.

Traditional organizations often deal with the issues of cognitive load and bias through the use of decision support systems tailored to managerial users $[1,2]$. These decision support systems could help a small group of people recognize problems, develop analytical syntheses, as well as identify and understand the consequences of potential solutions. Recently, some organizations have employed crowdsourcing as a strategy to support managerial decision making, problem-solving, and opportunity exploration by generating ideas, proposing solutions, or evaluating alternatives by the crowds [3]. In short, organizations have started to focus on how to leverage crowds to best support managerial requesters in formal decision-making. What is missing, however, is how to support the reasoning and decision processes of the crowd workers themselves.

Accurate reasoning does not always occur even when people are asked to address what might appear to be simple problems [4]. A variety of factors can undermine one's reasoning ability, including a general tendency to reach decisions using heuristics that although sometimes effective can also lead to systematic errors known as cognitive biases [5]. Additional challenges to accurate detection of reasoning issues include people's inflated and miscalibrated self-assessment due to the lack of metacognitive skill [6], and people's confidence in reasoning is frequently unrelated to the accuracy of their conclusion [7]. Although scholars have argued that seeking input from members of a group can potentially improve reasoning and the decisionmaking process [8], crowdsourcing platform designers still face the challenge of providing the appropriate reasoning and decision-making support to crowd workers. Moreover, recent research has revealed that crowds can be susceptible to some of the same cognitive biases as individuals [9].

In this paper, we present a novel approach to designing a crowdsourcing platform, i.e., TRACE, that can facilitate stigmergic coordination and communication and support crowds' reasoning and decision making. TRACE (Trackable Reasoning and Analysis for Collaboration and Evaluation) integrates crowdsourcing and Structured Analytic Techniques (STs) to strengthen intelligence analysts' reasoning and decision-making processes as well as improve the decisions that analysts and their customers make based on those processes. STs are "procedures for reducing the frequency and severity of error" [10]. Typical STs include devil's advocacy, brainstorming, and Analysis of Competing Hypotheses (ACH). Although STs have shown promise in various contexts, some debate their 
effectiveness, which varies depending on the specific type of problem and implementation of the procedure [11]. Moreover, STs are usually tested and implemented with individuals or teams by the Intelligence Community (IC) [12, 13]. As it stands, more research on organizational features that may strengthen STs' efficacy in large groups and crowd settings is needed to design crowdsourcing-based intelligence analysis system that can make collaborative reasoning more effective [10].

Moreover, intelligence analysts have long encountered issues with cognitive biases. Although IC studies point to significant problems with cognitive biases, solutions are rarely implemented or systematically monitored for efficacy. STs have been employed by the IC but rarely provide more than general process checks [11] and to date the STs used by the IC are not context sensitive, while analysts' instinctive reasoning are situated in context. This places a large cognitive burden on analysts to force the problem context to the structure of the technique. Crowd techniques offer an opportunity to support analysts' instinctual reasoning processes without adding significant cognitive load which not only detracts from the analysis and report-writing process, but also increases the amount of time needed to complete these processes.

In short, the IC faces a dilemma in how to best support analysts' reasoning and analysis. On the one hand, forcing them to use STs may improve the quality of their reasoning, but experience shows that STs' effects are contextual and debatable [11]. On the other hand, unstructured, laissez-faire approaches in intelligence analysis would increase the likelihood of analysts succumbing to various cognitive biases and thus impacting their analytic procedures and products. TRACE attempts to deal with this dilemma by combining the wisdom of the crowd and modified STs together.

\section{Theory}

The design of our TRACE system combines insights from the crowdsourcing literature with the conception of stigmergic coordination. The latter allows us to build our crowdsourcing system around the outcome of each crowd workers' labor rather than forced coordination, which can be time-consuming and burdensome.

\subsection{Crowdsourcing problems and tasks}

A crowdsourcing system is defined as a type of information system that leverages crowds to produce products and services for external or internal customers [14]. Since their emergence, crowdsourcing systems have been used by organizations or individual requesters to deal with different types of problems [ 15 , 16] ranging from knowledge discovery to distributed human intelligence tasks [15]. A common misconception about crowd-based problem solving is it only works well when dealing with non-complex problems [17, 18]. Recent studies, however, demonstrate that crowds can be leveraged to solve complex and collaborative tasks as well, such as writing fictional stories [19], generating competing ideas as well as proposals to manage climate change [20], and even contributing to making judgements and decisions [17].

Crowdsourcing systems rely on multiple organizational schemes. For example, Geiger et al. proposed a typology that includes four types of crowdsourcing systems: (1) crowd processing, which relies on homogenous contributions that meet predefined specifications and evaluation standards. Contributions are non-emergent which means they will come from individual crowd workers without any aggregation. Vizwiz is an example of this crowdsourcing system type [14]; (2) crowd rating, which relies on homogenous contributions from multiple workers. In other words, final output depends on the work of several individual crowd workers (e.g., an aggregated rating). The eBay reputation system as an example of this crowdsourcing type [14]; (3) crowd-solving, which relies on heterogeneous contributions from multiple workers (e.g., the generation of different and alternative solutions to a problem). In other words, worker contributions are independent of each other. 99designs.com is an example in this category [14]; and (4) crowd creation, which relies on heterogeneous and emergent contributions and results in a final output that is distinct from the inputs. According to Geiger et al., Wikipedia is an example in this category [14]. 
The foundation for the typologies of crowdsourcing above is a central organizational scheme which partitions a problem into sub-problems or divides a task into sub-tasks to be handled by crowd workers. Subsequently, there is a need to aggregate the resulting or mini-contributions in a meaningful way at appropriate junctures in the task completion process $[3,17,18]$. Partitioning a large problem into smaller units and then aggregating crowd contributions is not a trivial task. For sites such as Wikipedia that rely on crowd creation, the organization of crowd work sometimes leads to editing wars where participants repeatedly overwrite each other's work [21]. Sites relying on crowd ratings face issues with emerging and unruly labeling of work, spelling variations, the use of acronyms and synonyms, and varying terms approach within TRACE relies on stigmergic coordination which is defined as a process by which one individual affects the behavior of others through changes in the shared environment [24]. For example, ants follow scent trails to food previously found by other ants, thus assigning labor to the most promising sources. Organized collective action emerges from the interaction of individuals and the evolving environment rather than a shared plan. In other words, individuals build their own work on the traces left behind by other participants' contributions.

While stigmergy was formulated to explain the behavior of social insects following simple behavioral rules, it has also been invoked to explain classes of human activity. A bricklayer working on a wall may not need to explicitly coordinate his work with a

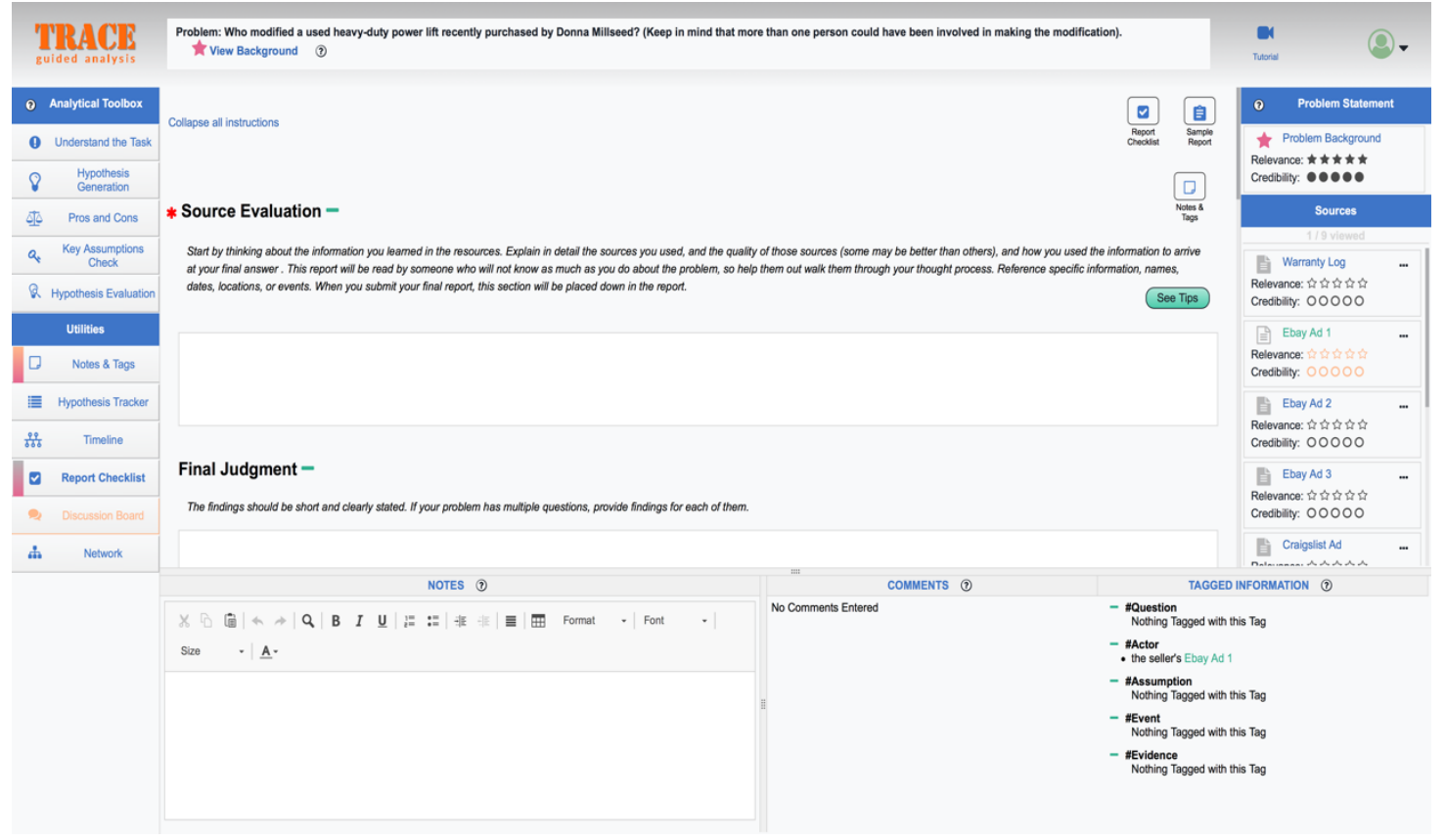

Figure 1. TRACE application layout. In the center of the screen is the report template that analysts use to compose their reports. To the right of the report is the source sidebar, which provides users with easy access to all of the sources they need to conduct their analysis. To the left of the report are TRACE's optional analytical tools and utilities.

used to describe overlapping phenomena [22, 23].

In short, it can be burdensome to develop an organizing scheme that effectively and appropriately partitions and aggregates crowdsourced work. This is particularly true for highly dynamic work environments such as the IC where the problems context and analytic tasks may change quickly.

\subsection{Stigmergic crowd coordination}

To overcome some of the challenges associated with partitioning and aggregating crowd work, our colleague before going on holiday. His fellow bricklayer can examine the patterns of bricks form the incomplete wall to determine where to place the next brick. As such, shared work itself can be a coordination mechanism. Christensen observed this type of coordination among architects, noting that their work is "partly coordinated directly through the material field of work... in addition to relying on second-order coordinative efforts (at meetings, over the phone, in emails, in schedules, etc.), actors coordinate and integrate their cooperative efforts by 
acting directly on the physical traces of work previously accomplished by themselves or others" [25].

The stigmergic form of crowd work allows participants to build on one another's contributions without explicit coordination among collaborators or the division of labor into discrete steps and responsibilities. But stigmergy does not mean that individuals uncritically mimicking the actions of other participants. If a large number of people work on the same problem, individuals will have a range of subtasks from which to select. We find these features make stigmergic coordination particularly promising for the IC because it may allow analysts to neutralize the impact of inaccurate, weak, or unreliable submissions (perhaps from sabotage) by giving them annotations to sources; (3) communicate with the other analysts about source annotations; (4) generate hypotheses; (5) debate the pros and cons of each hypothesis; (6) document any assumptions that could color the analysis; and (7) evaluate validity and alternative hypotheses.

The evaluation of information resources constitutes a critical task in evidence-based reasoning. Intelligence analysis is often a non-sequential threestep process of (1) sense-making, (2) critical evaluation, and (3) synthesis of available information. To help reasoners process and understand information from multiple sources, TRACE enables analysts to highlight, comment, and tag resources. For example, the tagging feature is designed to help reasoners identify and categorize critical pieces of information.

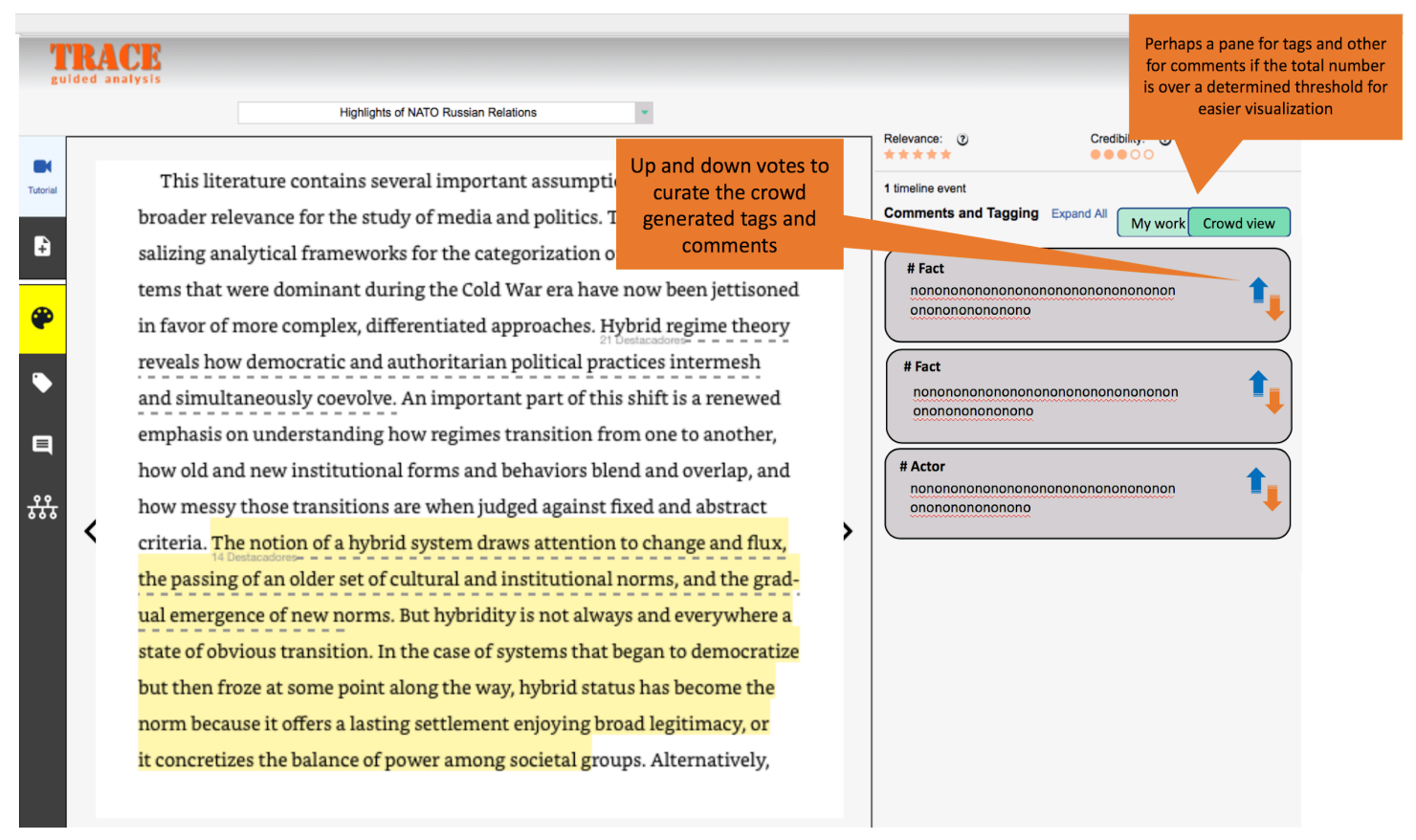

Figure 2. Mockup for the Tagging Tool in TRACE. The left side of the screen displays the source the user is currently viewing. The comments and Tagging pane is on the right side of the screen. This pane displays the user's tags for the source currently being viewed as well as the crowd's tags.

the ability to choose from a pool of contributions.

\section{The TRACE Approach}

TRACE is designed to enhance analysts' ability to reason and explain their judgment by combining modified STs with a stigmergic form of crowdsourcing. Figure 1 depicts the current version of the TRACE system which allows analysts to perform a variety of actions designed to enhance reasoning: (1) view different sources of information potentially relevant to the analytical task; (2) add tags and
It nudges them to think about actors, events, facts, and assumptions. The commenting feature is designed to aid sensemaking by helping analysts connect their opinions and insights to the available data. Additionally, these are the types of work that TRACE makes accessible to other analysts in the crowd, thus allowing them to extend their own analysis through stigmergic input from other's labor.

\subsection{Stigmergic awareness of others work}


At the core of the current TRACE approach to crowdsourcing-supported reasoning is the need to provide TRACE users with a stigmergic awareness of work contributed by others. In particular, TRACE intends to provide users with access to how others have used TRACE to complete key or challenging aspects of the reasoning process for the same case that the user is currently analyzing. Doing so offers two advantages: (1) Users may find particular parts of the reasoning process challenging or cognitively demanding, such as generating alternative hypotheses or identifying and considering key assumptions they have made which may influence their analyses. Whereas such challenges may prove insurmountable for someone working independently, access to crowd information allows users to build upon others' work those other information sources and thus mitigate anchoring bias [27].

Keeping with stigmergic coordination, the TRACE approach to crowdsourcing does not require users to share their work with others consciously. Instead, the TRACE software shares pivotal aspects of users' analysis (e.g., hypotheses, justifications, information resource evaluations, etc.) with other users. In this way, the TRACE approach to crowdsourcing does not interfere with users' natural reasoning processes or add additional burdens beyond what is already germane to the analytical task at hand.

As a proof of concept, we are currently implementing this stigmergic approach to TRACE's information resource evaluation and tagging tools. Figure 2 depicts the tagging tool in TRACE. The

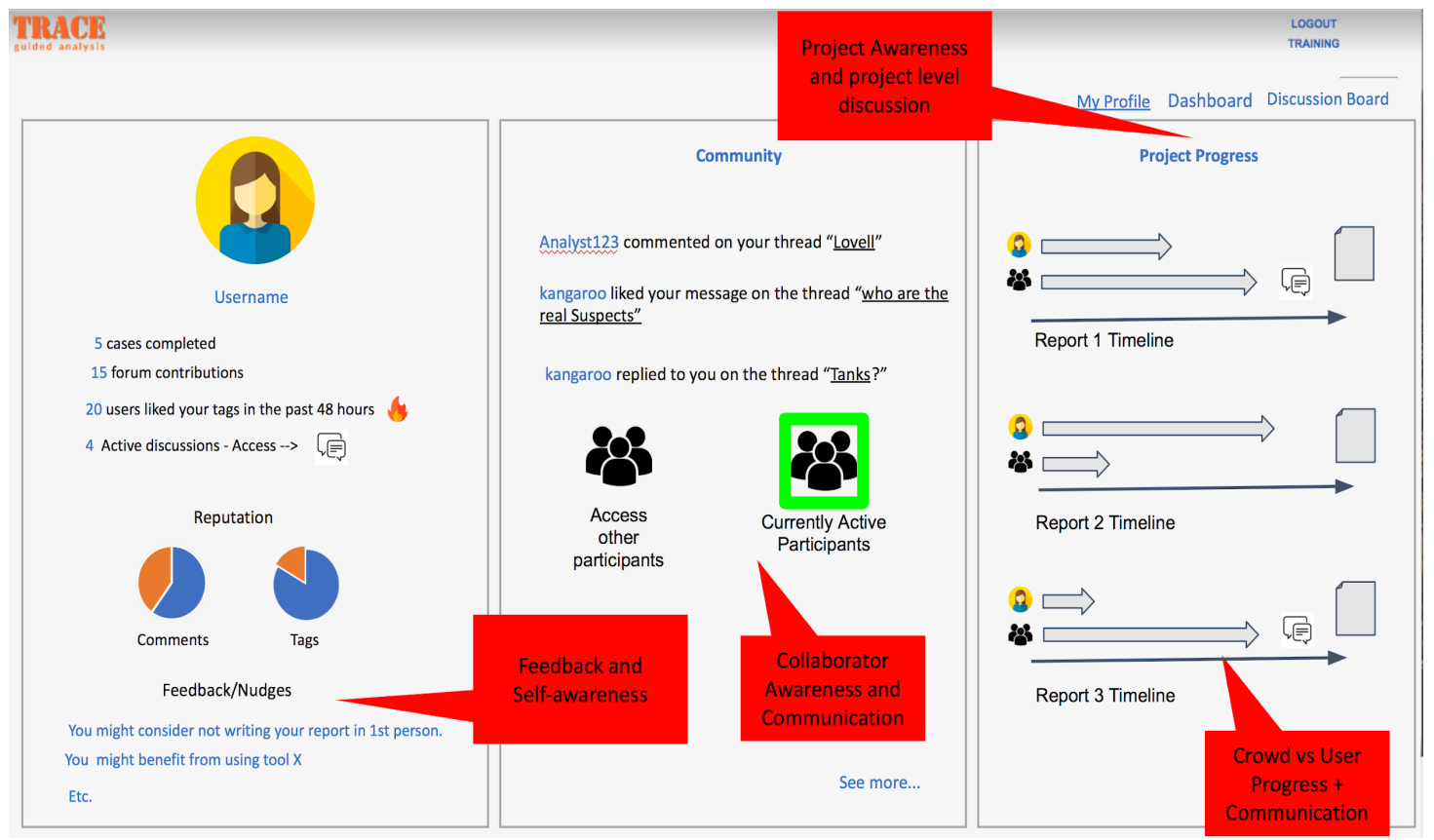

Figure 3. Stigmergic awareness of community in TRACE mockup

and incorporate these insights into their analysis. (2) Exposure to others' work may encourage a user to reconsider certain aspects of his or her reasoning process and thus help mitigate certain cognitive biases. For example, users' exposure to others' hypotheses and justifications may help them re-evaluate their final judgments and thus mitigate confirmation bias that seeks or interprets of evidence that are partial to existing beliefs, expectations, or a hypothesis in hand [26]. Additionally, users' access to how others have evaluated and annotated information sources may encourage users who pay an inordinate amount of attention to a particular piece of information while largely ignoring others to more carefully consider
TRACE software provides a suite of tools which help users evaluate resources necessary for their analysis. The resource evaluation feature allows users to rank the relevance and credibility of each piece of information based on a five-point scale and easily retrieve these rankings throughout their analysis. Additionally, TRACE includes a tagging tool that allows users to highlight and annotate particular pieces of information within a resource. The tagging tool provides four predetermined tags which can be used to identify and label "actors," "assumptions," "events," and "evidence." It also allows the user to create and utilize custom tags. Crowdsourced tagging can be seen as a type of collective intelligence, and a tag selected by the majority of crowd workers would embed a 
higher degree of CI than the other method [28]. Hence, the tagging feature not only supports users' initial sense-making process but also enables them to organize, sort, and then easily retrieve tagged data later in the analysis. Figure 2 above depicts the tagging tool in TRACE.

Crowdsourced tagging could also organize the annotations of the content in a structured and coordinated way. Traditionally, an obstacle that hinders the reasoning and comprehension of some content is that the user-generated annotations are poorly formatted, inconsistent, or ambiguous [22]. To tackle it, crowdsourced tagging could integrate a voting mechanism to select the most accepted and appreciated tags [23]. In future versions of the TRACE prototype, users will be able to access how others have utilized both the resource evaluation and tagging tools. The resource evaluation tool allows users to see how others evaluated the relevance and credibility of each information resource.

Additionally, the TRACE tagging tool will display such evaluative information as both an average score and a distribution of crowd worker scores across a five-point scale. TRACE users will also be able to view how other users have tagged pieces of information in the resources, including both annotated text and the labels tags applied to them. If a user finds a crowd worker's tag useful, they can choose to integrate it into their own work. The TRACE software treats imported tags as a favorable vote for that particular tag and uses these votes to determine the order in which to display all tags to users, with higher rated ones receiving precedent on the list.

\subsection{Stigmergic awareness of the community}

Prior research indicates that sociability, or the sense of being part of a valued community, is a crucial intrinsic motivator for active participants in a crowdsource project [29, 30, 31]. Keeping this in mind, the TRACE approach to crowdsourcing also provides users with an awareness of the community's collective work, including both what the crowd has done with the user's work as well as what the crowd has accomplished overall. This awareness gives participants a stigmergic sense of the work accomplished to date by all contributors. As an architect looks at an ongoing building project to assess the progress to-date, intelligence analysts will be able to gauge the progress of collective work by the contributions of the TRACE crowd.

To let users be aware of how others use their own work, the TRACE software will keep track of and notify users about the others' work imported from their contributions. For example, a user will be notified when other users import his or her tags into their analysis. This feature may remind users that they are not alone in this undertaking but are instead part of a community of reasoners. Additionally, this feature affords positive reinforcement in the form of feedback from others who found their work valuable. Prior research suggests displaying other community members' recent activities will strengthen a users' commitment to the collective [32]. For the current proof of concept, the goal is to implement the notifications feature for the tagging tool at this stage. If the preliminary findings are promising, we will explore the possibility of adding this stigmergic awareness feature to the other TRACE tools and tasks, such as hypotheses generation, assumption check, and the justification description.

Additionally, to make the user aware of the community's collective work, the TRACE software's home screen will include information about the crowds' aggregate contributions and accomplishments. This may include the number of hypotheses, assumptions, and tags the crowd has generated, the number of sources the crowd has evaluated, and the proportion of users who have completed their analyses. This information may motivate users to improve their analyses and further foster the sense that they are part of a broader community. Figure 3 depicts a mock-up design of this idea.

\subsection{Asynchronous communication}

In addition to the stigmergic aspects of others' work, the TRACE approach to crowdsourcing offers users a direct, asynchronous computer-mediated communication channel in the form of threaded forums. Asynchronicity provides many advantages for TRACE users. First, it allows users to meaningfully contribute and evaluate topic-related information without the need to be virtually co-present $[29,30]$. This mode of communication is preferable in crowd systems environments because it affords self-paced engagement with the tool [33], thus allowing crowd workers to post and respond to each other's comments at their convenience. It also fosters a sense of community among the crowd participants. Second, the permanence of asynchronous text-based communication allows TRACE to preserve group discourse, and thus enable analysis of transcripts with minimal risk of decontextualization or lost meaning $[30,31]$.

A thread in TRACE is a collection of forum posts on a particular topic. This provides users with a clear sense of the different topics under discussion and an easy way to access and participate in a particular topic of discussion. For example, users could utilize the 
forum to discuss tagging a source of information in order to better tag actors, evidence, and events

A thread could also be created to discuss a contingent topic. For example, users could deliberate tags or hypotheses generated that may not be agreed upon by the others. TRACE's threaded forums are currently case-specific, which means discussion related to one case would not interfere with discussions occurring among users working on a different case. This design prevents possible confusion associated with the availability of unrelated case content and different topics permeating into multiple cases. Thus, it could render the threaded discussion to be more focused within a specific case.

\section{Discussion and Conclusion}

In this paper, we have presented the TRACE system, an application that supports reasoning and decisionmaking by combining structured analytic techniques and a stigmergic form of crowdsourcing. We are designing TRACE to help intelligence analysts to improve the quality of their reasoning. We expect that the system will not only mitigate analysts' cognitive biases in reasoning but also help produce higher quality analytic products that outline the reasoning and analysis processes in ways that others can readily comprehend. If TRACE works as expected, it should be of interest beyond the IC and enable a range of crowdsourcing efforts such as crowdsourcing-based product design, collective action for politics, and decision support system.

The stigmergic approach should be particularly useful for crowdsourcing projects that place a high cognitive burden on participants. Our stigmergic approach within TRACE will allow individuals to draw on other users' contributions and apply it to not only complement their efforts but also contribute contrasting viewpoints to the work of others and see trends in crowd solutions, thus allowing the individual analysts to approach tasks more critically. Our approach differs from traditional STs in the IC that tend to be both time-consuming and cognitively burdensome for analysts.

Besides the implication to the IC, the proposed stigmergic approach could also contribute to the scholarship of crowdsourcing and information system research more broadly. Stigmergic coordination and communication among the crowds may potentially facilitate various crowdsourcing processes which often rely on a central actor to partition and later synthesize the crowd work into a coherent product. The stigmergic crowdsourcing approach such as that in TRACE, in contrast, would allow individuals to develop their own analysis and with the benefit of having access to other crowd workers' effort.

The current TRACE system serves as a promising platform for future experimentation and iterative improvements. Moving forward, we plan to examine the impact of our stigmergic approach to structured techniques on common cognitive biases that tend to emerge during intelligence analysis. Doing so will allow us to explore whether the proposed crowd solution minimizes common biases or introduces new ones into the process. For example, as several prior studies have found [9, 23], we could deliberately expose TRACE users in an experimental setting to crowd work that is intended to anchor them on a particular piece of information or evaluation to determine if and how the anchoring effect may impact analysts in a stigmergic crowd setting.

Second, a stigmergic approach encourages users to pay close attention to their work as well as the work of others. If users pay more attention to contributions, they would be inclined to engage in conversations about them, which introduces the need to consider the best way to facilitate productive communication. Also, such a stigmergic approach raises the question about where crowd communication should take place in order to promote productivity. To answer this question, we hope to compare dialogue facilitated by a standalone asynchronous discussion forum interface with one embedded in specific analytical tasks such as the tagging tool. A direct form of communication would allow crowd workers to discuss specific tags, clarify points, or reconcile disagreements as they do their tagging work.

Finally, we plan to conduct experiments to understand how more structured taxonomies may improve communications within the stigmergic environment. For example, we would like to know how the addition of structured taxonomists (individuals who maintain and enhance the taxonomy language) may help to improve communication, understanding, and ultimately reasoning.

The main contribution of this paper has been to discuss how a combination of stigmergic crowdsourcing and structured analytic techniques can be used to facilitate reasoning and decision support in crowd settings characterized by cognitively demanding tasks. The proposed system gives analysts the freedom to draw on the work by others without taking away their agency or pre-determining their analysis process. Although we are currently still refining the TRACE system, our ambitious plans to conduct more rigorous experimentation will generate promising empirical findings that fill current gaps in the scholarly literature on collaboration and reasoning 
as well as inform the design of future crowdsourcing platforms.

\section{References}

[1] Sprague Jr, R. H. (1980). A framework for the development of decision support systems. MIS quarterly, 126.

[2] Shim, J. P., Warkentin, M., Courtney, J. F., Power, D. J., Sharda, R., \& Carlsson, C. (2002). Past, present, and future of decision support technology. Decision support systems, 33(2), 111-126.

[3] Chiu, C. M., Liang, T. P., \& Turban, E. (2014). What can crowdsourcing do for decision support? Decision Support Systems, 65, 40-49.

[4] Frederick, S. (2005). Cognitive reflection and decision making. Journal of Economic Perspectives, 19, 25 42 .

[5] Gilovich, T., Griffin, D., \& Kahneman, D. (Eds.). (2002). Heuristics and biases: The psychology of intuitive judgment. New York: Cambridge University Press.

[6] Kruger, J., \& Dunning, D. (1999). Unskilled and unaware of it: How difficulties in recognizing one's own incompetence lead to inflated self-assessments. Journal of Personality \& Social Psychology,77, 1121-1134.

[7] Shynkaruk, J.M. \& Thompson, V.A. (2006). Memory \& Cognition, 34, 619-632.

[8] Kahneman, D., \& Tversky, A. (1972). Subjective probability: A judgment of representativeness. Cognitive psychology, 3(3), 430-454.

[9] Eickhoff, C. (2018, February). Cognitive Biases in Crowdsourcing. In Proceedings of the Eleventh ACM International Conference on Web Search and Data Mining (pp. 162-170). ACM.

[10] Heuer, R. J. (2008, March). Taxonomy of structured analytic techniques. In International Studies Association Annual Convention (pp. 1-6).

[11] Coulthart, S. J. (2017). An Evidence-Based Evaluation of 12 Core Structured Analytic Techniques. International Journal of Intelligence and CounterIntelligence, 30(2), 368-391.

[12] Artner, S. J., Girven, R., \& Bruce, J. B. (2016). Assessing the Value of Structured Analytic Techniques in the US Intelligence Community. Rand Corporation.
[13] Primer, A. T. (2009). Structured Analytic Techniques for Improving Intelligence Analysis. CIA Center for the Study of Intelligence.

[14] Geiger, D., Rosemann, M., Fielt, E., \& Schader, M. (2012). Crowdsourcing Information Systems-Definition Typology, and Design.

[15] Brabham, D. C. (2013). Crowdsourcing. John Wiley \& Sons, Inc..

[16] Gadiraju, U., Kawase, R., \& Dietze, S. (2014, September). A taxonomy of microtasks on the web. In Proceedings of the 25th ACM conference on Hypertext and social media (pp. 218-223). ACM.

[17] Kittur, A., Smus, B., Khamkar, S., \& Kraut, R. E. (2011, October). Crowdforge: Crowdsourcing complex work. In Proceedings of the 24th annual ACM symposium on User interface software and technology (pp. 43-52). ACM.

[18] Kittur, A., Nickerson, J. V., Bernstein, M., Gerber, E., Shaw, A., Zimmerman, J., ... \& Horton, J. (2013, February). The future of crowd work. In Proceedings of the 2013 conference on Computer supported cooperative work (pp. 1301-1318). ACM.

[19] Kim, J., Sterman, S., Cohen, A. A. B., \& Bernstein, M. S. (2018). Mechanical novel: Crowdsourcing complex work through reflection and revision. In Design Thinking Research (pp. 79-104). Springer, Cham.

[20] Malone, T. W. (2011). Solving Climate Change with Crowdsourcing. MIT Sloan Management Review.

[21] Sumi, R., \& Yasseri, T. (2011, October). Edit wars in Wikipedia. In Privacy, Security, Risk and Trust (PASSAT) and 2011 IEEE Third International Conference on Social Computing (SocialCom), (pp. 724-727). IEEE.

[22] Peters, I., \& Weller, K. (2008). Tag gardening for folksonomy enrichment and maintenance. Webology, 5(3), $1-18$.

[23] Corey Jackson, Kevin Crowston, Carsten Østerlund, Mahboobeh Harandi (2018 forthcoming). Folksonomies to support coordination and coordination of folksonomies. ECSCW.

[24] Dipple, A., Raymond, K., \& Docherty, M. (2014). General theory of stigmergy: Modelling stigma semantics. Cognitive Systems Research, 31, 61-92.

[25] Christensen, L. R. (2008, November). The logic of practices of stigmergy: representational artifacts in architectural design. In Proceedings of the 2008 ACM conference on Computer supported cooperative work (pp. 559-568). ACM. 
[26] Nickerson, R. S. (1998). Confirmation bias: A ubiquitous phenomenon in many guises. Review of general psychology, 2(2), 175.

[27] Furnham, A., \& Boo, H. C. (2011). A literature review of the anchoring effect. The Journal of Socio-Economics, 40(1), 35-42.

[28] Yi, K. (2012). Harnessing collective intelligence in social tagging using Delicious. Journal of the Association for Information Science and Technology, 63(12), 24882502.

[29] Brabham, D. C. (2010). Moving the crowd at Threadless: Motivations for participation in a crowdsourcing application. Information, Communication \& Society, 13(8), 1122-1145.

[30] Goh, D. H., \& Lee, C. S. (2011). Perceptions, quality and motivational needs in image tagging human computation games. Journal of Information Science, 37(5), 515-531.

[31] Nov, O., Anderson, D., \& Arazy, O. (2010, April). Volunteer computing: a model of the factors determining contribution to community-based scientific research. In Proceedings of the 19th international conference on World wide web (pp. 741-750).

[32] Ren, Y., Kraut, R., Kiesler, S., \& Resnick, P. (2012). Encouraging commitment in online communities. Building successful online communities: Evidence-based social design, 77-124.

[33] Hull, D.M. and Saxon, T.F. 2009. Negotiation of meaning and co-construction of knowledge: An experimental analysis of asynchronous online instruction. Computers \& Education. 52, 3 (Apr. 2009), 624-639. 